\title{
How not to count the health benefits of family planning
}

\author{
Jacob Zionts, Joseph Millum
}

Clinical Center Department of Bioethics, National Institutes of Health, Bethesda, Maryland, USA

\section{Correspondence to} Mr Jacob Zionts, Clinical Center Department of Bioethics, National Institutes of Health, Bethesda, Maryland, USA; jmzionts@gmail.com

Received 16 June 2021 Accepted 26 November 2021

\section{ABSTRACT}

Several influential organisations have attempted to quantify the costs and benefits of expanding access to interventions - like contraceptives - that are expected to decrease the number of pregnancies. Such health economic evaluations can be invaluable to those making decisions about how to allocate scarce resources for health. Yet how the benefits should be measured depends on controversial value judgments. One such value judgment is found in recent analyses from the Disease Control Priority Network (DCPN) and the Study Group for the Global Investment Framework for Women's and Children's Health. Noting the decrease in the number of pregnancies expected to result from providing access to family planning, DCPN and the Study Group claim that a substantial benefit of such interventions is averting the stillbirths and child deaths that would have resulted from those pregnancies. We argue that health economic analyses should not count such averted deaths as benefits in the same way as saved lives. First, by counting averted stillbirths and child deaths as a benefit but not counting as a cost the lives of babies who survive, DCPN and the Study Group implicitly commit themselves to antinatalism. Second, this method for calculating the benefits of family planning interventions implies that infertility treatments are harmful. Determining how potential people should be treated in health economic analyses will require grappling with population ethics.

\section{INTRODUCTION}

Health systems around the world operate under conditions of scarcity. Not all interventions that would benefit people in the populations they serve can be funded, so difficult choices must be made about where to allocate limited resources. Making these decisions in systematic and justifiable ways requires data on the costs and the expected benefits of different possible health interventions.

One important set of interventions that healthcare systems may provide are reproductive, maternal, newborn and child health (RMNCH) interventions. Some RMNCH interventions, such as contraceptives, provide individuals with the means to prevent conception and are therefore predicted to reduce the number of pregnancies in a population. Some others, such as treatment of chlamydia and gonorrhoea, instead reduce individuals' risks of infertility.

Two influential research groups-the Study Group for the Global Investment Framework for Women's and Children's Health (the Study Group) and the Disease Control Priorities Network (DCPN)-have published comprehensive models of the health and economic benefits of scaling up $\mathrm{RMNCH}$ interventions in low-income and middleincome countries (LMICs). ${ }^{12}$ For the most part, the
Study Group and DCPN's analyses of the costs and health benefits of RMNCH interventions look just like other health economic analyses that are used as inputs to decision-making. But something curious happens when they present the health benefits of reproductive health interventions that decrease the number of pregnancies among their targeted populations, such as increasing access to family planning and contraceptives.

One health benefit from a decrease in pregnancies is a drop in maternal mortality. But the decrease in the number of pregnancies, the Study Group and DCPN argue, has another, more substantial benefit: a huge reduction in the number of stillbirths and child deaths. Some of these stillbirths and child deaths are prevented because wider birth spacing is associated with healthier children. ${ }^{3}$ However, the majority of the prevented newborn and child deaths that the Study Group and DCPN calculate as benefits of family planning are averted by preventing the decedents from ever existing. ${ }^{12}$

In this paper we critically assess the claim that evaluations of family planning interventions should count averting potential stillbirths and child deaths through non-existence as benefits in the same way they count other health benefits. We argue that they should not. As Hilary Greaves has previously argued, there are significant differences between saving an existing fetus or child's life through preventing a source of mortality like HIV and averting a potential fetus or child's death through non-existence. ${ }^{4}$ If deaths averted through nonexistence are to be counted as benefits, then this requires both substantial normative argument and the inclusion of a set of beneficiaries much wider than just third-trimester fetuses and children who die young.

The paper progresses as follows. Section two describes the findings of the Study Group and DCPN. Section three argues that in choosing to count averted stillbirths and child deaths as benefits but not count long, healthy lives that are also averted as costs, the Study Group and DCPN implicitly commit themselves to some sort of antinatalist position. Section four argues that the Study Group and DCPN's method for calculating the benefit of reproductive healthcare interventions that predictably reduce the number of pregnancies or births implies that infertility treatments are harmful or otherwise problematic. Section five offers a diagnosis. We argue that the Study Group and DCPN's mistaken reasoning is proximately caused by treating the size of a population itselfnumbers of people qua numbers of people-as a health problem. It is ultimately caused by their treatment of questions about bringing people into 
existence, which are problems in population ethics, in the same way as ethical questions about existing people.

\section{CALCULATIONS OF THE BENEFITS OF FAMILY PLANNING}

The Study Group is coordinated by the WHO and the Partnership for Maternal, Newborn \& Child Health. Their Global Investment Framework for Women's and Children's Health makes the case for 'how investment in women's and children's health will secure high health, social, and economic returns'. ${ }^{1}$ They model the costs and benefits of expanding coverage of six packages of RMNCH interventions in 74 countries. These countries have been selected for their high burden of maternal and child mortality; combined, they have a population of 4.9 billion and account for over $95 \%$ of maternal and child deaths worldwide. The health interventions include access to family planning, maternal and newborn health interventions (eg, post-abortion care, skilled birth assistance, folic acid supplementation), malaria and HIV prevention and treatment, immunisations and child healthcare (eg, oral rehydration therapy, antibiotics for dysentery).

According to the Study Group's model, a 'high-coverage scenario' would entail spending an additional US\$5 per person per year ( $\sim$ US\$30 billion per year). They estimate it would prevent 5 million maternal deaths, 147 million child deaths and 32 million stillbirths by 2035 . Roughly half of the child deaths prevented can be chalked up to promotive, preventative and curative health services (eg, oral rehydration therapy and zinc for diarrhoea treatment). These prevented deaths are lives saved. The rest result from scaled up family planning, which reduces the number of pregnancies, and so the number of births, and so the number of deaths. These prevented deaths are deaths averted. The Study Group writes:

\begin{abstract}
Of the 147 million child deaths prevented, 78 million (53\%) would be deaths averted from scaling up family planning and 69 million (47\%) would be lives saved from scaling up promotive, preventive and curative health services. Expanding access to contraception will be a particularly effective investment, accounting for half of the deaths averted, at small cost ( $4 \%$ of additional intervention-specific cost 2013-35). ${ }^{1}$
\end{abstract}

The DCPN project is funded by the Bill and Melinda Gates Foundation and its Disease Control Priorities volumes are published by the World Bank Group. Their Reproductive, Maternal, Newborn and Child Health volume identifies 61 interventions that span reproductive health, maternal and newborn health, and child health. They model the costs and effects of expanding coverage of each intervention to $90 \%$ of the population of 75 high-burden countries (the Study Group's 74 plus one). Like the Study Group, DCPN singles out the impact of increased access to family planning:

\begin{abstract}
The reproductive health package is particularly important for providing contraceptive services. Addressing $90 \%$ of unmet need in 2015 would reduce annual births by almost 28 million, which would consequently prevent 67000 maternal deaths; 440000 neonatal deaths; 473000 child deaths; and 564000 stillbirths from avoided pregnancies. ${ }^{2}$
\end{abstract}

\section{IMPLYING ANTINATALISM}

An intervention that reduces the number of people born in a population will reduce the number of deaths in that population because those who are never born cannot die. The Study Group and DCPN's calculation of the health benefits of family planning considers a subset of these potential people: those who, in the absence of the intervention, would have been born and then quickly died. They count preventing that particular subset of potential people from coming into existence as improving the health of the population. But the great majority of the potential people whose existences are prevented by contraceptive use would not die early deaths. Many would live long, largely healthy lives. What do the Study Group and DCPN have to say about this larger group of potential people? Are what they consider the deleterious effects of potential people meeting early deaths counteracted by the positive value of potential people living long, healthy lives?

Interestingly, the latter group is not mentioned: averting the lives of the potential people who, in the absence of the analysed interventions, would have been born and then lead flourishing lives is not counted as a downside of the interventions. At best then, the Study Group and DCPN place no value on creating someone who will lead a long, healthy life. However, they negatively value lives that quickly end. This implies that the Study Group and DCPN treat being born as either neutral or bad.

Consider what this asymmetry in the way that the Study Group and DCPN treat creating lives means for their health economic analyses of interventions that affect the number of pregnancies. If an intervention brings a single life with negative value into existence, such as a child who dies as a neonate, then the calculation of that intervention's impact on potential people will always come out negative. That would be the case even if the same intervention brought millions of flourishing lives into existence, because those lives are assigned at best neutral (zero) value. Since any time an individual is brought into existence there is some chance that they will die very early, the expected value of any intervention that affects the number of pregnancies will be negative. The Study Group and DCPN's position is therefore functionally antinatalist. In other words, they have at least tacitly bought into the position that bringing people into existence is, on balance, always bad. ${ }^{5}$

Antinatalism is a minority and controversial position. ${ }^{6}$ We therefore think it is unlikely that the Study Group and DCPN are actually committed to the view. It is more likely that they did not realise the functionally antinatalist implications of treating averted lives as either a health loss to a population or as neither loss nor gain. If the Study Group and DCPN are in fact committed to an antinatalist position, they should make that commitment explicit and defend it. On the other hand, if the Study Group and DCPN are not antinatalists, then they need to re-formulate their method for calculating the health benefits of interventions that affect the number of pregnancies in a population.

\section{DEVALUING INTERVENTIONS THAT REDUCE INFERTILITY}

A second reason to object to the Study Group and DCPN's position is that, consistently applied, it would imply that certain interventions have a negative impact because they treat or avert infertility. Recall that for the Study Group and DCPN, one mechanism by which stillbirths and child deaths are prevented is reduced pregnancies. Reproductive health interventions that reduce the number of pregnancies are thereby characterised as beneficial partially in terms of their impact on the number of stillbirths and child deaths. Following the same logic, interventions that increase the number of pregnancies should be regarded as harmful_or at least negatively valued-insofar as they thereby increase the number of stillbirths and child deaths. 
That has potentially serious consequences for health economic analyses of interventions that address infertility.

Consider the Guttmacher Institute's estimate that all women living in LMICs who are infected with chlamydia, gonorrhoea, trichomoniasis and syphilis-the four major sexually transmitted infections (STIs)—could be cured for US $\$ 2.3$ billion. $^{7}$ They regard this as a highly cost-effective use of healthcare funds. One of the major health benefits of treatment is reduction in infertility: the Guttmacher Institute claims that treating all women in LMICs infected with chlamydia and gonorrhoea alone would prevent 3 million cases of infertility caused by those STIs. ${ }^{7}$ Notably absent in their calculation of benefit is any consideration of the intervention's impact on potential peopledespite the fact that reducing instances of unwanted infertility is likely to increase the number of pregnancies among the treated population. But if the Guttmacher Institute applied the Study Group and DCPN's thinking here, then health economic analyses of the intervention might come out negative. That is because investing money on averting preventable instances of infertility, thereby decreasing involuntary childlessness and increasing the number of pregnancies, would potentially lead to an increase in the number of stillbirths and child deaths.

Devaluing interventions that address infertility is highly problematic for a number of reasons. We mention just one here, which we believe is the most important. Reproductive justice, as conceptualised by its contemporary advocates, centres around the right to have children, the right not to have children and the right to raise children in a safe and healthy environment. ${ }^{89}$ Crucially, the reproductive justice movement places equal value on the right to have children and the right not to have children, grounding both in the principle of reproductive autonomy. By devaluing health interventions that address involuntary childlessness caused by preventable infertility, the Study Group and DCPN undermine one side of the reproductive autonomy coin-the right to have children. That unfortunate fact should resonate with them, since both appear motivated by a desire to uphold and advance the right to reproductive healthcare. In fact, the Study Group's analysis explicitly acknowledges 'the intrinsic value of health and its role in meeting basic human rights'. ${ }^{1}$ DCPN, for its part, grounds all of its work in a human rights-based approach, arguing that one of the primary reasons for investing in increased access to reproductive healthcare is meeting basic 'human rights and equity' concerns. ${ }^{2}$

\section{A POSSIBLE DIAGNOSIS}

There is a mistake in reasoning made by the Study Group and DCPN that helps explain why they end up arguing that we can prevent the deaths of some fetuses, newborns and young children by preventing them from ever existing. The mistake is easily replicable, so it is worth explicitly identifying and addressing it.

The proximate source of the mistake is that the Study Group and DCPN treat population itself-numbers of people qua numbers of people-as a health problem. As we have noted, for the Study Group and DCPN, one mechanism by which stillbirths and child deaths are prevented is reduced pregnancies. If someone does not become pregnant, then they do not give birth, and so there is no risk that they give birth to a baby that dies. This logic can be extended beyond RMNCH. All else equal, a reduction in the number of pregnancies will also have the effect of reducing the number of young adults who die in road traffic accidents, develop mental disorders and so on, simply because the individuals who would experience these health events will not come into existence. Indeed, all else equal, any incremental reduction in the size of a population will almost always lead to a reduction in the population's total burden of disease. This is a function of the fact that almost everyone experiences some ill health in their life. Removing a potential container of disease from a heap of containers will typically reduce the expected total amount of disease.

The implication of this reasoning is that if asked to choose between two possible futures that only differed in the size of their populations, we should prefer the future with the smaller population. Critically, we would be pushed to make that choice not because the future with the smaller population contained healthier people, but just because it contained fewer unhealthy people. Total burden of disease would be lower, but that would not entail a decrease in the average burden of disease.

Treating population itself as a health problem is problematic. We believe that the Study Group and DCPN correctly presuppose that health systems are and ought to be invested in reducing disease burden. However, we contend that within that credo lies an implicit condition: reductions in the disease burden should come from health interventions that target sources of morbidity and mortality affecting people who exist or will exist, rather than merely decreasing the number of people who will come into existence. Without that condition, the focus of health systems might shift, in part, away from health and toward population reduction-foci that are not obviously complementary.

That brings us to our diagnosis of the mistake: the Study Group and DCPN treat decisions that affect who and how many people are born in the same way as they treat decisions that do not affect who is born. But ethically these are quite different types of decisions. The latter, which encompasses most moral philosophy, considers the ethics of decisions that affect individuals who exist or will exist independent of those decisions. For example, neither choice available to the agent in the famous Trolley Problem directly affects who comes into existence. ${ }^{10}$ The choice at hand determines which existing individuals will survive or be killed.

The former set of decisions is the subject matter of an area of moral philosophy called 'population ethics'. Population ethics analyses decisions that affect who is born and how many people are born. Policymakers deciding whether to increase access to assisted reproductive technologies are engaged in population ethics: their decision affects both who is born and also how many people are born. The practice of population ethics often involves making holistic evaluations about the relative goodness and badness of possible futures that differ primarily in population size and quality of life. For example, it is a live question within the field whether and when increasing the size of a population while decreasing the average lifetime well-being of its members is wrongful.

The evaluation of $\mathrm{RMNCH}$ interventions is complicated because many of them have predictable and substantial effects on both individuals who will exist independent of the intervention and on which individuals come into existence. When the Study Group and DCPN combine lives saved and deaths averted through non-existence, they elide this distinction. But, as Greaves notes, this elision means that saving a 1-year-old from dying so that they go on to live another 60 years is evaluated the same as stopping the 1-year-old from ever coming into existence. ${ }^{4}$ Furthermore, as we noted above, insofar as the Study Group and DCPN engage in population ethics, they include only some of the effects on who will come to exist-they count young deaths averted but not the lives and deaths of those who would have lived much longer. There are multiple competing views within population ethics, but most would require us to 
start with a full accounting of the quality and quantity of lives under different allocation scenarios.

It is important to be explicit about the limited nature of our diagnosis. We are not claiming that the Study Group and DCPN's mistake was caused by their failure to adhere to a particular view within population ethics. Rather their mistake was caused by not recognising that they had entered into a realm that requires grappling with a particular kind of moral thinking.

\section{CONCLUSION}

The Study Group and DCPN claim that we can prevent stillbirths and child deaths by preventing the decedents from ever existing. They then count those averted deaths as a health benefit of reproductive interventions that reduce the number of pregnancies. We have argued that this position is mistaken: there are philosophically and practically significant differences between averting stillbirths and child deaths through non-existence and other ways to prevent mortality. As currently described, the Study Group and DCPN's methodology implies a view that is functionally antinatalist. Moreover, the Study Group and DCPN's methodology devalues reproductive healthcare interventions that combat infertility and might shift the attention of health systems toward population reduction. We argued that the Study Group and DCPN make those mistakes because they treat population itself as a health problem and fail to recognise that population ethics involves different ethical considerations than the considerations used to evaluate decisions that only affect existing people.

We draw two sets of conclusions. The first has to do with what analysts should do now. To start, health economic analyses of interventions that affect the number of viable pregnancies should count stillbirths and child deaths averted through non-existence only if (a) there is a full accounting of the interventions' effects on who comes into existence and how many people come into existence (ie, including people who do not die young); and (b) a justification for how the benefits stemming from potential people are counted relative to those coming from existing people. If that cannot be done, then health economic analyses should simply not count stillbirths and child deaths averted through non-existence as benefits of $\mathrm{RMNCH}$ interventions. They should, of course, continue to count the lives of existing people those interventions save.

The second set of conclusions has to do with directions for future research. Determining how potential people should be treated in health economic analyses will require grappling with population ethics. Critically, that would be the case even if the Study Group and DCPN took a different approach. Population ethics must be considered in any situation where decisions are being made about which people and how many people come into existence. At this point, we do not claim that there is a particular view that the Study Group and DCPN should adopt-that is a topic for further research.

We are now in the process of canvassing methods that avoid the inconsistencies described here, meaningfully consider population ethics and usefully measure the benefits of interventions that affect the number of viable pregnancies. One possibility would be to follow Greaves' lead and search for an appropriate 'value function that simply evaluates states of affairs'. ${ }^{4}$ Another possibility would be to modify and then extend Leigh Senderowicz's operationalised concept of 'contraceptive autonomy'. ${ }^{11}$ And a third possibility would be to avoid using summary measurements of health or well-being altogether and instead adopt intermediate measurements like 'couple years of protection'. ${ }^{3}$ Each of these options has pros and cons that still need to be explored.

\section{Twitter Jacob Zionts @jzionts}

Acknowledgements Thank you to Agneska Bloch, Annette Rid, David Wasserman Jacob Barrett, Sarah Raskoff, and our anonymous reviewers for providing substantive and insightful comments on our drafts. And thank you to the all participants at the American Society for Bioethics and Humanities conference and Institute for Bioethics and Health Humanities' Reproductive Ethics Conference who provided such useful feedback. Finally, thank you to the NIH Clinical Center Department of Bioethics for supporting our work.

Contributors JZ and JM jointly conceived of, wrote and edited the paper. JZ wrote the first draft; IZ and JM jointly approved the final draft. JZ is the guarantor.

Funding The authors have not declared a specific grant for this research from any funding agency in the public, commercial or not-for-profit sectors.

Disclaimer The views expressed are those of the authors. They do not represent the positions or policies of the National Institutes of Health, the Department of Health and Human Services or the US Government.

Competing interests None declared.

Patient consent for publication Not applicable.

Ethics approval This study does not involve human participants.

Provenance and peer review Not commissioned; externally peer reviewed.

Data availability statement Data sharing not applicable as no data sets generated and/or analysed for this study. N/A.

\section{REFERENCES}

1 Stenberg $\mathrm{K}$, Axelson $\mathrm{H}$, Sheehan $\mathrm{P}$, et al. Advancing social and economic development by investing in women's and children's health: a new global investment framework. Lancet 2014:383(9925):1333-54.

2 Black R, Laxminarayan R, Temmerman M. Reproductive, Maternal, Newborn, and Child Health. Disease Control Priorities. Vol 2. 3rd edn. Washington, DC: World Bank, 2016.

3 Stover J, Hardee K, Ganatra B. Interventions to Improve Reproductive Health. In: Sternberg K, Axelson H, Sheehan P, eds. Reproductive, maternal, newborn, and child health. disease control priorities. 3rd edn. Washington, DC: World Bank, 2016: Vol 2. 95-114.

4 Greaves H. Against "the Badness of Death.". In: Gamlund E, Tollef Solberg C, eds. Saving people from the harm of death. New York: Oxford University Press, 2019: 189-202.

5 Benatar D. Better Never to Have Been: The Harm of Coming into Existence. New York: Oxford University Press, 2006.

6 Benatar D, Wasserman D. Debating procreation: is it wrong to reproduce? New York: Oxford University Press, 2015.

7 Sully E, Biddlecom A, Darroch J. Adding It Up: Investing in Sexual and Reproductive Health 2019. New York: Guttmacher Institute, 2020.

8 SisterSong. Reproductive Justice [Internet]. Cited 2021 April 19. Available: https:// www.sistersong.net/reproductive-justice

9 Zavella P. The Movement for Reproductive Justice: Empowering Women of Color through Social Activism. New York: NYU Press, 2020.

10 Thomson JJ. Killing, Letting die, and the trolley problem. Monist 1976;59(2):204-17.

11 Senderowicz L. Contraceptive autonomy: conceptions and measurement of a novel family planning indicator. Stud Fam Plann 2020;51(2):161-76. 